\title{
Sportverletzungen an der Schulter
}

\author{
Dirk Maier, Martin Jaeger, Wolfgang Köstler, Norbert P. Südkamp
}

\section{Zusammenfassung}

Die Zahl der jährlichen Unfälle beim Sport wird in Deutschland auf 1,52 Millionen geschätzt [1]. Sportunfälle machen inzwischen 25 - $30 \%$ aller Unfälle aus. 7,7\% der Verletzungen entfallen hierbei auf die Schulter. Bezogen auf die Körperregion tritt die Gelenkluxation mit 57\% am häufigsten an der Schulter auf. Der folgende Artikel möchte einen Überblick über häufige Sportverletzungen an der Schulter geben. Aufgrund des großen Umfanges der Thematik kann der Überblick nicht vollständig sein. Die Schwerpunkte der Arbeit liegen auf Verletzungen des Akromioklavikulargelenkes (ACG), der Schulterluxation und der Schulterinstabilität. ACG-Verletzungen vom Typ Rockwood I und II werden mit guten Ergebnissen konservativ therapiert. Die Gesamtergebnisse sprechen bei der Rockwood-III-Verletzung für die konservative Therapie. Es ist jedoch eine differenzierte Analyse im konkret vorliegenden Fall erforderlich, um die für den Patienten beste Therapieoption zu finden. Rockwood-IV- und -V-Ver- letzungen werden operativ versorgt. Es herrscht nach wie vor keine Einigkeit bei der Wahl des OP-Verfahrens zur Versorgung akuter ACG-Luxationen. Die Therapieentscheidung bei der Schultererstluxation hängt entscheidend vom Lebensalter des Patienten ab. Die operative Stabilisierung der Schulter muss die zugrunde liegende strukturelle Pathologie berücksichtigen. Im Falle einer begleitenden Fraktur des Tuberculum majus ist eine Rezidivinstabilität äußerst selten $\mathrm{zu}$ beobachten. Vielmehr bestimmt das Ausmaß der Frakturdislokation das weitere therapeutische Vorgehen.

\section{Sports-Related Injuries of the Shoulder}

In Germany, the number of accidents per year in sports is estimated at about 1.5-2 million. Accidents in sports account for approximately $25-30 \%$ of all accidents. The shoulder is affected in $7.7 \%$ of the cases. The highest proportion of joint dislocations (57\%) occurs at the shoulder joint. This article describes common sports injuries at the shoulder joint. However, the extent of the subject does not allow a complete review. Focus is placed on injuries of the acromioclavicular joint (AC joint), dislocation of the shoulder and shoulder instability. Treatment of Rockwood I and II injuries is non-surgical. Overall results after Rockwood III injuries favour non-surgical therapy when compared to surgical treatment. However, a differentiated analysis is required to find the optimal therapeutic option for each individual patient. Rockwood IV and V injuries require operative treatment. To date, there is no consensus on a standard surgical procedure for the acute repair of AC joint dislocations. The therapy for primary dislocation of the shoulder strongly depends on the age of the patient. Operative stabilisation of the shoulder must take the underlying structural pathology into consideration. Recurrent shoulder instability is rarely seen with concomitant fracture of the greater tuberosity. Therapy is rather determined by the extent of fracture dislocation.

\section{Luxation des Akromioklavikular- gelenkes}

\section{Epidemiologie}

Die Inzidenz der Akromioklavikulargelenkluxation (ACG-Luxation) wird mit ca. 3-4\% der Gelenkluxationen angegeben. Männer sind häufiger betroffen als Frauen, das Verhältnis wird auf $5: 1$ bis 10:1 geschätzt. Es handelt sich dabei um eine typische Sportverletzung.

OP-JOURNAL 2008; 24: 144-149

(c) Georg Thieme Verlag KG Stuttgart • New York DOI 10.1055/s-2008-1038952
In der Statistik der Sportverletzungen stellt sie die dritthäufigste Verletzung dar [1].

\section{Pathogenese}

In der Regel geht ein direktes Anpralltrauma voraus (bsp. Fahrradsturz auf die laterale Schulter bei angelegtem Oberarm), wobei das Akromion eine Relativbewegung nach kaudal durchführt und die laterale Klavikula nach kranial luxiert. Bei dem weitaus selteneren indirekten Unfallmechanismus mit Sturz auf den ausgestreckten Arm kommt es durch den Humeruskopf zu einer Krafteinwirkung von unten auf das Akromion. Das Akromion bewegt sich relativ nach kranial. Hierbei kann es auch zur sehr seltenen ACG-Luxation Typ Rockwood VI kommen. In Abhängigkeit der Schwere des Traumas kommt es zur Zerrung oder Zerreißung der Bandstrukturen zwischen Processus coracoideus und Klavikula (Lig. trapezoideum, Lig. conoideum) bzw. zwischen Akromion und lateraler Klavikula (Lig. acromioclaviculare). Bei höhergradigen Luxationen rupturieren auch die umgebenden Faszien des M. deltoideus und M. trapezius.

\section{Klassifikation}

Die Klassifikation nach Rockwood hat sich in der Klinik zur Beschreibung von Verletzungen des Akromioklavikularge- 


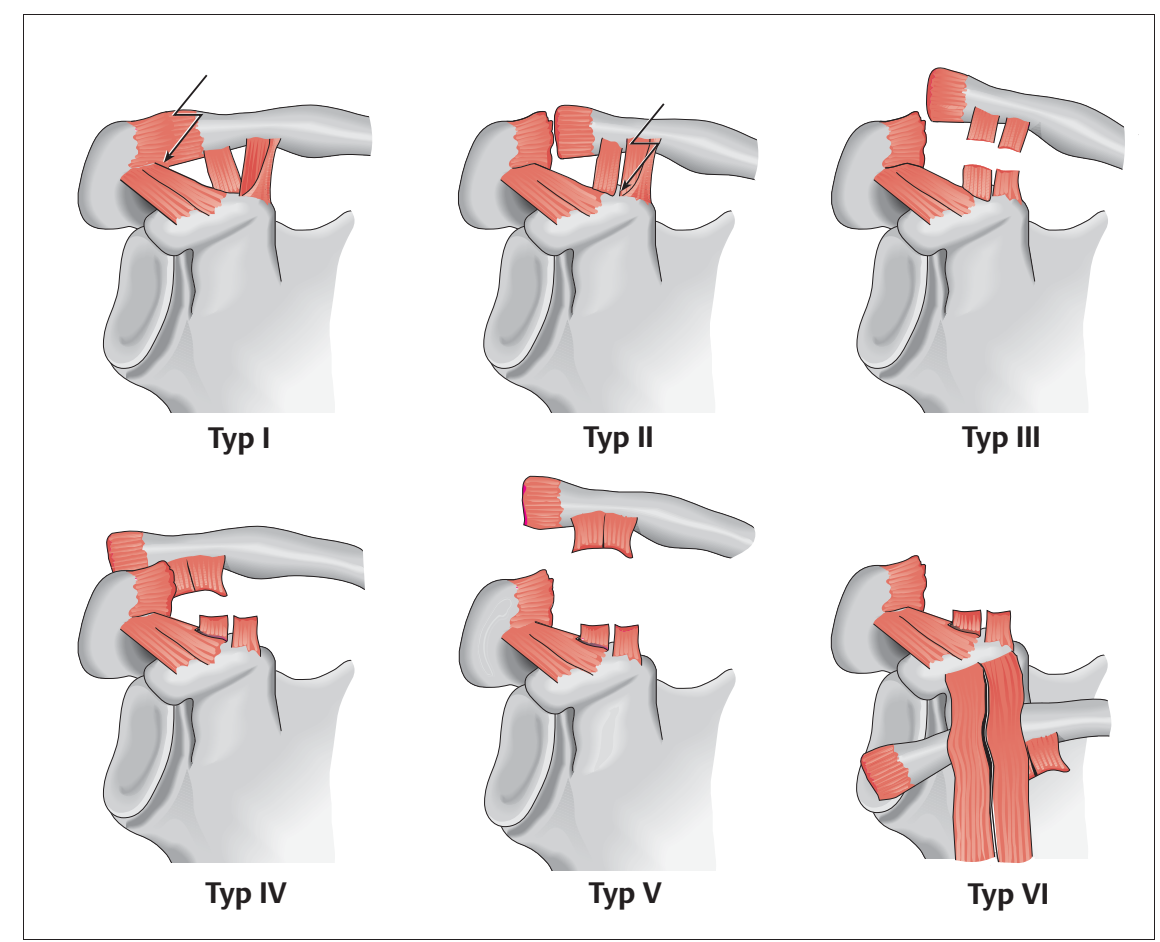

Abb. 1 Klassifikation der Akromioklavikulargelenksverletzungen nach Rockwood.
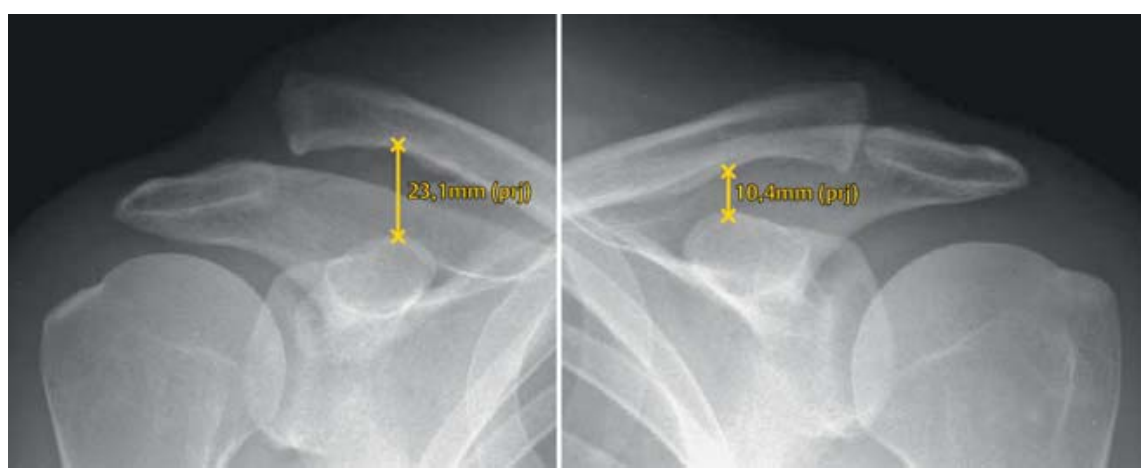

Abb. 2 Bestimmung des korakoklavikulären Abstandes bei Rockwood-V-Verletzung in gehaltenen ACG-Aufnahmen.

lenkes etabliert. Aufgrund ihrer genaueren Einteilung hat sie die früher verwendete Klassifikation nach Tossy abgelöst. Rockwood unterscheidet 6 Typen, wobei die Typen I-III nach Rockwood gemäß der Klassifikation nach Tossy ebenso den Typen I-III entsprechen (Abb.1). Die Rockwood-Klassifikation wird in den meisten klinischen Studien verwendet und hat somit auch konkreten Einfluss auf die Therapieauswahl.

\section{Diagnostik}

Klinisch imponiert eine Schwellung und ggf. ein Hochstand der lateralen Klavikula in Relation zum Akromion. Richtigerweise handelt es sich um einen Tiefstand der Skapula in Relation zur ne Aufnahmen (sogenannte „Wasserträgeraufnahmen“) im Seitenvergleich zuverlässige Aussagen über den funktionellen Bandstatus zu (Abb. 2). Es handelt sich hierbei um Röntgenaufnahmen beider Akromioklavikulargelenke im a.-p. Strahlengang (gleiche Projektion) unter Längszug der Arme. Panoramaaufnahmen sind aus strahlenhygienischen Gründen obsolet. Es ist darauf zu achten, dass die Gewichte (10 kg) an Schlaufen frei an den Handgelenken des sitzenden oder stehenden Patienten hängen und dieser die Gewichte nicht in der Hand festhält. Außerdem sollte eine ausreichende Analgesie erfolgen. Bei den gehaltenen Aufnahmen wird auf jeder Seite der korakoklavikuläre Abstand bestimmt. Anhand des relativen Verhältnisses dieser Distanzen lässt sich die Klassifikation nach Rockwood vornehmen.

Bei klinischem Verdacht auf eine Rockwood-IV-Verletzung empfehlen wir die Durchführung einer transaxillären Aufnahme bzw. einer Alexander-Aufnahme (funktionell), um eine posteriore Dislokation der Klavikula nachweisen zu können.

Suspekt für eine Rockwood-IV-Verletzung ist auch eine Vergrößerung des ACGelenkspaltes in der a.-p. Projektion oder in der Zanca-Aufnahme. In der Praxis wird eine Rockwood-IV-Verletzung leicht übersehen, sodass bei klinischem Verdacht auch die weiterführende Diagnostik mittels CT zur Evaluation des Verletzungsausmaßes hilfreich sein kann.

\section{Therapie}

Typ-I- und -II-Verletzungen können mit guten Ergebnissen konservativ therapiert werden. Da es sich um eine bandstabile Verletzung handelt, kann die Therapie schmerzadaptiert und frühfunktionell erfolgen. Eine Ruhigstellung im Gilchrist-Verband ist in der Regel nicht erforderlich, kann aber ggf. für einen Zeitraum von wenigen Tagen erfolgen. Die krankengymnastische Beübung kann frühzeitig unter bedarfsweise verabreichter Analgesie begonnen werden. Physikalische Maßnahmen und ggf. Lymphdrainage können ergänzend eingesetzt werden. Schweres Heben, Liegen auf der betroffenen Seite sowie eine schulterbelastende Sportart sollten abhängig vom Heilverlauf für 3-6 Wochen vermieden werden. Die Therapie von Typ-III-Verletzungen wird in der Literatur kontrovers diskutiert [2]. Der 
Evidenzgrad der meisten Studien zu diesem Thema ist leider gering, es existieren nur sehr wenige Studien mit einem Evidenzgrad II oder III. Die vorliegenden prospektiven, randomisierten und kontrollierten Studien ergaben leichte Vorteile der konservativen Therapie gegenüber der operativen Therapie [3]. Die Vorteile der konservativen Therapie lagen in der niedrigeren Komplikationsrate, kürzeren Rehabilitationsphase und kürzeren Dauer der Arbeitsunfähigkeit bei vergleichbarem funktionellen $\mathrm{Er}$ gebnis. Die Datenlage berücksichtigt allerdings nicht die aktuellen Möglichkeiten der operativen Versorgung und basiert zumeist auf OP-Techniken, die heute nicht mehr verwendet werden.

Im Einzelfall kann sicherlich die operative Versorgung der Rockwood-III-Verletzung indiziert sein und die für den Patienten individuell bessere Therapieoption darstellen. Dies trifft vor allem für Patienten mit einem sehr hohen funktionellen Anspruch und schulteraktive Sportler (z. B. Werfer) zu [4].

Möglicherweise ist auch eine differenzierte Betrachtung der Rockwood-IIIVerletzung hilfreich, so variiert das Verletzungsausmaß (Zunahme des korakoklavikulären Abstandes von 25-100\%) erheblich innerhalb der Entität. Die ACG-Luxationen Typ IV bis VI werden operativ versorgt. Es findet sich in der Literatur eine Vielzahl von Operationsmethoden zur Versorgung der ACG-Luxation. Dies ist Ausdruck dafür, dass sich bis heute noch keine Operationsmethode allgemein anerkannt durchsetzen konnte. Die Prinzipien der Stabilisierung des ACG lassen sich nach verschiedenen Gesichtspunkten einteilen. So kann die Fixierung intra- oder extraartikulär erfolgen. Es werden akromioklavikuläre und korakoklavikuläre Stabilisierungen sowie Kombinationen dieser beiden Verfahren unterschieden. Häufig eingesetzte Verfahren sind die Reposition und Transfixation des ACG mittels K-Drähten oder Pins mit oder ohne Rekonstruktion der korakoklavikulären Bänder. Die Reposition des ACG kann auch mit einer Bosworth-Schraube gehalten werden, welche die laterale Klavikula am Processus coracoideus fixiert. In jüngerer Zeit liegt der Fokus auf arthroskopisch-assistierten Verfahren, die eine Rekonstruktion der ligamentären Anatomie zum Ziel haben. Vielversprechende Frühergebnisse liegen für die arthroskopisch-assistierte transkorakoidale, transklavikuläre Rekonstruktion

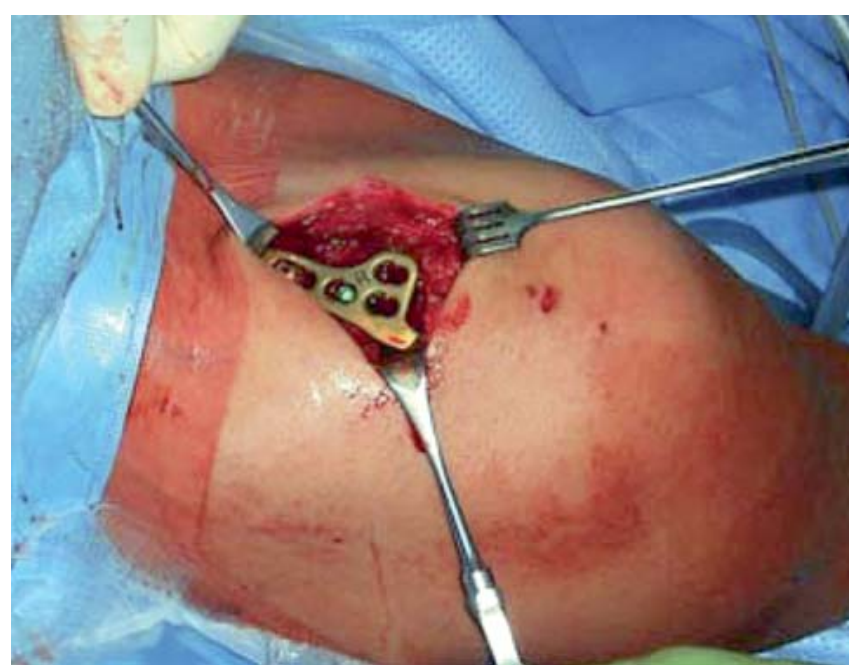

Abb. 3 Intraoperativer Situs nach Implantation der winkelstabilen $\mathrm{AO}$ Hakenplatte.

der korakoklavikulären Bänder mittels Tight-Rope ${ }^{\circledR}$ (Fa. Arthrex) vor. Es bleibt abzuwarten, ob sich diese Verfahren mittel- bis langfristig bei einer größeren Anzahl von Patienten bewähren können. Zur Versorgung chronischer ACG-Luxationen kann die Resektion der lateralen Klavikula (Mumford, Gurd 1941) ggf. in Kombination mit einem Transfer der korakoklavikulären Bänder (Weaver, Dunn 1972) erfolgen. Auch für die Versorgung chronischer Verletzungen existieren arthroskopisch-assistierte Verfahren, die mit einer Grazilis- oder Semitendinosus-Sehnenplastik augmentiert werden. Die ACG-Arthrodese wird heute nur noch sehr selten durchgeführt.

Balser beschrieb 1976 die erste Plattenversorgung einer ACG-Luxation. Diese sogenannte „Hakenplatte“ wurde im Laufe der Zeit im Design verändert und kontinuierlich weiterentwickelt. In unserer Klinik setzen wir die winkelstabile 3,5-mm-LCP (AO-Hakenplatte, Fa. Synthes) ein. Dieses Implantat liefert reproduzierbare, gute langfristige klinische Ergebnisse nach Versorgung akuter ACG-Luxationen. Es kann auch zur Versorgung chronischer Verletzungen eingesetzt werden, allerdings ist dann eine Augmentationsplastik z. B. mittels Grazilis- oder Semitendinosussehne erforderlich.

\section{OP-Technik der AO-Hakenplatte}

Der Patient ist auf dem Rücken gelagert, der Oberkörper ist ca. $30^{\circ}$ angehoben. Präoperativ muss überprüft werden, ob eine ausreichende röntgenologische Darstellung in 2 Ebenen (a.-p., axial) möglich ist. Es erfolgt ein ca. $5 \mathrm{~cm}$ langer
„Säbelhiebzugang“ auf Höhe des ACG. Nach stumpfer Präparation der Faszie wird selbige im Längsverlauf der lateralen Klavikula inzidiert. Bei RockwoodV-Situationen tritt die häufig deperiostierte laterale Klavikula spontan nach oben. Die Reposition des ACG erfolgt unter makroskopischer und radiologischer Kontrolle (Bildwandler). Das ACG wird temporär mittels K-Draht transfixiert. Die winkelstabile AO-Hakenplatte (meist 2-Loch) wird mit ihrem Haken dorsal des ACG unter das Akromion eingehängt und mit 2 winkelstabilen, bikortikalen Schrauben am Klavikulaschaft fixiert (Abb.3). Es ist auf eine exakte Ausrichtung des Implantates zu achten. Der Haken sollte hierbei plan der Akromionunterfläche anliegen (Abb.4). Bei Instabilität in a.-p. Richtung (Rockwood IV) muss der K-Draht ggf. belassen werden, um eine Dislokation nach posterior zu verhindern. Es erfolgen die Rekonstruktion des akromioklavikulären Kapsel-Band-Apparates, der Faszienverschluss sowie die Subkutan- und Hautnaht. Die rupturierten korakoklavikulären Bänder werden von uns bei dieser OP-Technik nicht primär rekonstruiert, sondern heilen indirekt durch Adaptation der Bandstümpfe.

\section{Nachbehandlung}

Postoperativ wird die Schulter schmerzabhängig für wenige Tage immobilisiert und einer frühfunktionellen Nachbehandlung zugeführt. Die Gesamtabduktion ist dabei für 3 Monate auf $90^{\circ}$ limitiert, die glenohumerale Abduktion kann somit voll ausgenutzt werden. Die Implantatentfernung des K-Drahtes erfolgt ggf. nach 6 Wochen, die der Platte nach 3-6 Monaten. Hierbei muss darauf 


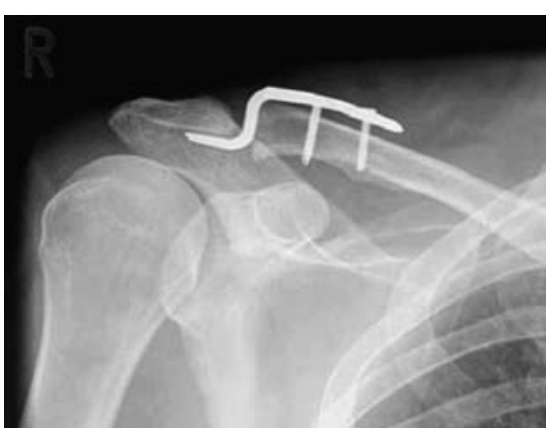

Abb. 4 Röntgenkontrolle nach Implantation der winkelstabilen $\mathrm{AO}$-Hakenplatte.

geachtet werden, den akromioklavikulären Kapsel-Band-Apparat möglichst gering zu schädigen und wieder anatomisch zu rekonstruieren. Die Nachbehandlung ist frühfunktionell ohne Bewegungslimitation möglich. Für einen Zeitraum von 6 Wochen nach Implantatentfernung der Platte sollte auf Gewichtsbelastungen und Überkopfsport verzichtet werden.

\section{Luxation des Humeroglenoidal- gelenkes}

\section{Epidemiologie}

Hovelius et al. [5] untersuchten die Ergebnisse nach primärer anterior-inferiorer Schulterluxation in Schweden. Die Arbeitsgruppe veröffentlichte 2008 die 25-Jahres-Ergebnisse ihrer Multicenterstudie. Die Inzidenz der Luxation betrug in der Bevölkerung zwischen 18 und 70 Jahren ca. 1,7\%, wobei Männer ca. 3-mal häufiger betroffen waren als Frauen. In Dänemark liegt die Inzidenz bei 17/100 000 in der Gesamtbevölkerung. Überträgt man diese Daten auf die Bundesrepublik Deutschland, ist hierzulande von einer Inzidenz von 13600 Fällen bezogen auf die Gesamteinwohnerzahl auszugehen. Im Kindes- und Adoleszentenalter beträgt die jährliche Luxationsrate ca. 4,5\%. Im Alter von unter 14 Jahren beträgt die Inzidenz von Schulterluxationen dagegen nur ca. 0,5\%. Die Inzidenz nimmt weiterhin mit steigendem Lebensalter deutlich ab. Die Inzidenz von Rezidivluxationen des Schultergelenkes ist deutlich altersabhängig. Nach Rowe et al. [6] beträgt sie nach konservativer Therapie für 10- bis 20-Jährige $94 \%$, für 20 - bis 30 -Jährige $79 \%$ und für 30 - bis 40 -Jährige $50 \%$. Diese Daten müssen vor dem Hintergrund der Ergebnisse der Studie von Hovelius et al. kritisch diskutiert werden. Die Rezidivrate 25 Jahre nach Erstluxation lag bei den 12-40-jährigen Patienten bei ca. $50 \%$. $43 \%$ der Patienten hatten keine erneute Luxation, $7 \%$ gaben ein einmaliges weiteres Luxationsereignis an, 27\% wurden operativ stabilisiert und $22 \%$ waren persistierend instabil mit weiteren Luxations- oder Subluxationsereignissen. Ein signifikanter Risikofaktor für ein Rezidiv war das jüngere Lebensalter. Die 3- bis 4-wöchige Schulterimmobilisation in Innenrotation senkte die Rezidivrate nicht signifikant. Auch konnte kein Unterschied zwischen Sportlern und Nichtsportlern festgestellt werden.

\section{Pathogenese}

Die glenohumerale Luxation lässt sich nach ihrer Luxationsrichtung unterscheiden. Ca. 95\% aller Luxationen sind anterior-inferiore Luxationen, bei denen sich der Humeruskopf nach medial subglenoidal verschiebt. Häufig handelt es sich um Sportunfälle mit direktem Anpralltrauma (z. B. Sturzereignis). Die Luxation kann auch durch ein indirektes Trauma (z.B. Krafteinwirkung auf den abduzierten, extendierten und außenrotierten Arm) erfolgen. Deutlich seltener treten mit ca. 3\% die posterioren Luxationen sowie die „Raritäten“ der superioren und zentralen Luxation sowie der Luxatio erecta auf. Die posterioren Luxationen werden häufig im Rahmen eines zerebralen Krampfanfalles oder eines Stromunfalles, seltener durch indirekte Krafteinwirkung auf den adduzierten, extendierten und innenrotierten Arm beobachtet. Die Luxatio erecta entsteht durch ein Hyperabduktionstrauma, wobei das Akromion als Hypomochlion dient und der Humeruskopf nach anterior-inferior herausgehebelt wird. Der Luxation ohne adäquates Trauma (habituelle Luxation) liegt eine anlagebedingte Pathologie im Bereich der Weichteile, Knochen oder Muskeln zugrunde (z.B. Kapsel-Band-Hyperlaxizität, knöcherne Dysplasie, neuromuskuläre Erkrankung).

\section{Klassifikation}

Im klinischen Alltag haben sich vor allem die Klassifikationen nach Bayley bzw. nach Gerber bewährt, da sie die Pathogenese der Instabilität berücksichtigen und somit auch Einfluss auf die Therapie der Wahl nehmen. Bayley (2002) beschreibt neben einer traumatischen und atraumatischen Genese als Ursache für die Schulterinstabilität die muskuläre Dysbalance als wichtigen ätiopathologischen Faktor in der Polar
Group III. Darunter versteht man eine gestörte Funktion bzw. Innervation der Rotatorenmanschetten- und Schultergürtelmuskulatur, die zu einer glenohumeralen Instabilität führt. Nachweisbar pathologische EMG-Muster können hier eine koordinierte muskuläre Führung des Kopfes in der Pfanne verhindern. Jede Form der Schulterinstabilität lässt sich auf einem gedachten Dreieck, welches zwischen den 3 Polen gespannt ist, einordnen.

Polar Group I: traumatisch, strukturell

- signifikantes Trauma

- oft Bankart-Läsion

- gewöhnlich unilateral

- keine muskuläre Dysbalance

Polar Group II: atraumatisch, strukturell

- kein Trauma

- struktureller Schaden des Gelenkes

- kapsuläre Dysfunktion

- keine muskuläre Dysbalance

- nicht selten bilateral

Polar Group III: habituell, nicht strukturell

- kein Trauma

- keine strukturellen Schäden des Gelenkes

- kapsuläre Dysfunktion

- muskuläre Dysbalance

- oft bilateral

\section{Diagnostik}

Die Diagnose einer Schulterluxation lässt sich häufig bereits klinisch stellen. Der Patient hält die betroffene Schulter in Schonhaltung, wobei der betroffene Arm vom gesunden Arm unterstützend gehalten wird. Der betroffene Arm befindet sich in der Regel in einer leicht abduzierten und innenrotierten Stellung. Sowohl die aktive als auch die passive Beweglichkeit der Schulter sind schmerzbedingt stark eingeschränkt oder aufgehoben. Bei der häufigen anterior-inferioren Luxation palpiert sich unter dem prominent erscheinenden Akromion eine leere Mulde. Wichtig ist die rasche Überprüfung des neurovaskulären Status. Insbesondere muss die Funktion des N. axillaris (Sensibilität im Bereich der Schulterkappe über M. deltoideus) sowie des Armplexus (Fingersensibilität und -motorik) untersucht werden. Eine Röntgenuntersuchung der Schulter in 2 Ebenen (true a.-p. und outlet) ist im Rahmen der Notfalldiagnostik ausreichend. Bei einer korrekt projizierten True-a.-p.-Aufnahme findet sich eine Doppelkontur, die aus dem ante- 
rior-inferior lokalisierten Humeruskopf und dem Glenoidrand gebildet wird. In der Outlet-Aufnahme zeigt sich ebenso eine anterior-inferiore Verlagerung des Humeruskopfes. Bei beiden Aufnahmen ist zusätzlich auf eine begleitende Fraktur zu achten. Eine weitere bildgebende Diagnostik ist im luxierten Zustand in der Regel nicht indiziert. Lediglich bei Luxationsfrakturen sowie bei verhakten, geschlossen nicht reponierbaren Luxationen kann eine CT hilfreich sein zur Planung der rasch durchzuführenden Notfalloperation. Unmittelbar nach dem Repositionsmanöver einer glenohumeralen Luxation ist eine standardisierte klinische und bildgebende Diagnostik obligat. Insbesondere ist die erneute Überprüfung des neurovaskulären Status wichtig. Die Röntgendiagnostik nach Reposition hat immer in 2 Ebenen zu erfolgen (true a.-p. und outlet). Es ist unbedingt darauf zu achten, dass der glenohumerale Gelenkspalt in der Truea.-p.-Ebene uneingeschränkt einsehbar ist. Nur so lässt sich eine regelrechte glenohumerale Artikulation nachweisen.

\section{Therapie}

Zur Reposition der anterior-inferioren Schulterluxation empfehlen wir weichteilschonende Techniken ohne direkte Krafteinwirkung im Bereich der Axilla. Bei der Repositionstechnik nach Matsen befindet sich der Patient in Rückenlage, wobei das Ellenbogengelenk zur Entlastung des $\mathrm{M}$. bizeps und des M. brachioradialis $90^{\circ}$ flektiert ist. Es wird ein gleichmäßiger axialer Zug am Arm über einen Gurt oder ein Tuch aufgebaut, welches zwischen dem Chirurgen und dem Unterarm des Patienten platziert wird. Simultan wird durch eine Hilfsperson ein konstanter Zug in Gegenrichtung ausgeübt, über ein Tuch, das um den Thorax des Patienten geschlungen ist. Wichtig ist zu beachten, dass das Tuch für den Gegenzug nicht in der Axilla positioniert ist und somit als Repositionshindernis für den Humeruskopf wirken könnte. Durch den konstanten axialen Zug kommt es zur weichteilschonenden Reposition. Evtl. kann eine geringe Außenrotationsbewegung oder ein Hilfszug nach lateral die Reposition unterstützen. Sehr schonend gelingt die Reposition auch in Bauchlage. Da eine Ruhigstellung in Innenrotation die Rezidivrate nicht günstig beeinflussen kann, halten wir diese nicht über einen längeren Zeitraum für erforderlich. Häufig dient sie lediglich der Sicherung des Repositionsergebnisses beim analgo- sedierten Patienten. Die Innenrotationsruhigstellung kann rasch zugunsten der funktionellen Nachbehandlung aufgegeben werden. Neuere Studien weisen auf einen günstigen Einfluss einer Außenrotationsruhigstellung auf die Rezidivrate hin [7]. Der ventrale Kapsel-Labrum-Komplex reponiert sich durch die Weichteilspannung anatomischer und scheint suffizienter zu verheilen. Insbesondere jüngere Patienten unter 30 Jahren scheinen von einer Außenrotationsruhigstellung über einen Zeitraum von 3 Wochen zu profitieren.

\section{Indikation zur OP}

Die Empfehlung zur Stabilisierung der Schulter nach Erstluxation ist abhängig von der zugrunde liegenden strukturellen Pathologie und dem Lebensalter des Patienten.

Liegen Begleitpathologien wie z.B. eine knöcherne Bankart-Läsion, Glenoidfraktur, Rotatorenmanschettenruptur, SLAPLäsion oder Kapsel-Läsion (HAGL-Läsion) vor, so beeinflusst dies die Therapiefindung. Meist müssen diese strukturellen Begleitpathologien chirurgisch addressiert werden. Im Rahmen der Diagnostik sollte ein MRT der Schulter sowie ggf. eine CT durchgeführt werden, um solche Befunde nicht zu übersehen. Bei der unkomplizierten Erstluxation ohne eine solche Begleitpathologie hängt die Empfehlung zur Stabilisierung entscheidend vom Lebensalter des Patienten ab. In der Studie von Hovelius et al. [5] lag der Anteil der operativ stabilisierten oder instabilen Schultern 25 Jahre nach Erstluxation bei den 12 -16-jährigen Patienten bei $60 \%$, bei den 23-25-Jährigen bei ca. $40 \%$ und bei den 34-40-Jährigen bei ca. $20 \%$. In Kenntnis dieser Daten sollte eine ausführliche Beratung und Aufklärung des Patienten über die Prognose der Erkrankung erfolgen. Sollte die Therapie primär konservativ erfolgen, so ist die Indikation zur operativen Stabilisierung bei persistierender Instabilität oder Reluxation gegeben. Bei jüngeren Patienten kann sowohl die primär operative Stabilisierung als auch ein konservativer Therapieversuch durchgeführt werden.

Offene Stabilisierungsverfahren gelten aufgrund der vorliegenden Langzeitergebnisse und der niedrigen Rezidivrate noch als der Goldstandard. Klassische Verfahren sind hierbei die offene Refixation des Kapsel-Labrum-Komplexes nach Bankart (Bankart-OP) oder ver- schiedene Techniken des Kapselshifts (z.B. T-Shift nach Neer) [8]. Arthroskopische Stabilisierungsverfahren werden zunehmend bevorzugt, da sie die Rezidivraten der offenen Verfahren zumindest mittelfristig nachweisbar erreichen (0-10\%) und mit einer deutlich geringeren Zugangsmorbidität assoziiert sind [9]. So resultiert die Ablösung der Subskapularissehne bei den offenen Verfahren neben einer postoperativen Limitation der Rotation langfristig auch mit einer funktionell relevanten Subskapularisinsuffizienz in ca. einem Fünftel der Patienten [10].

\section{OP-Technik der arthroskopischen Schulterstabilisierung}

Wir führen die Operation in Seitenlagerung durch. Der zu operierende Arm wird in einem Doppelarmhalter mit $6 \mathrm{~kg}$ Längszug und $2 \mathrm{~kg}$ Vertikalzug unter sterilen Bedingungen aufgehängt, abgewaschen und abgedeckt. Nach Abschluss der diagnostischen Arthroskopie des glenohumeralen Gelenkes über den dorsalen Standardzugang werden unter Sicht (outside-in) zunächst ein $8,25 \mathrm{~mm}$ anteriorer Standardzugang unmittelbar kranial der Subskapularissehne sowie ein 5,75 $\mathrm{mm}$ anterior-superiorer Zugang oberhalb der langen Bizepssehne angelegt. Die Insertion von transparenten $\mathrm{Zu}$ gangskanülen mit Gewinde hat sich zum Schutz des Weichteiles und zur Vermeidung von Gewebebrücken bewährt. Es erfolgt zunächst die Mobilisierung des ventralen Labrums und der Kapsel bis der ventrale Komplex von der darunterliegenden Subskapularismuskulatur gelöst ist und nach Ablassen des Spüldruckes spontan auf Glenoidhöhe „aufschwimmt“. Hierbei bewährt sich insbesondere bei einem weit medial im Sinne einer ALPSA-Läsion fehlverheilten Kapsel-Labrum-Komplex das Wechseln der Optik in das anterior-superiore Portal, um die Übersicht zu erhöhen. Anschließend wird der Glenoidhals von anhängendem Weichteilgewebe débridiert. Hierzu eignen sich unterschiedlich gebogene Bankart-Raspeln, es kann aber auch ein Weichteil-Shaver eingesetzt werden. Sinnvoll ist es ferner, den ventralen Anteil des Gelenkknorpels sparsam mit einer arthroskopischen Kürette zu débridieren, um hier eine gute Einheilung des Labrums zu ermöglichen. Zur Fixierung des mobilisierten KapselLabrum-Komplexes eignen sich Nähte, die mit Suture-Lassos eingebracht werden können. Die Fäden werden temporär im anterior-superioren Portal „ge- 


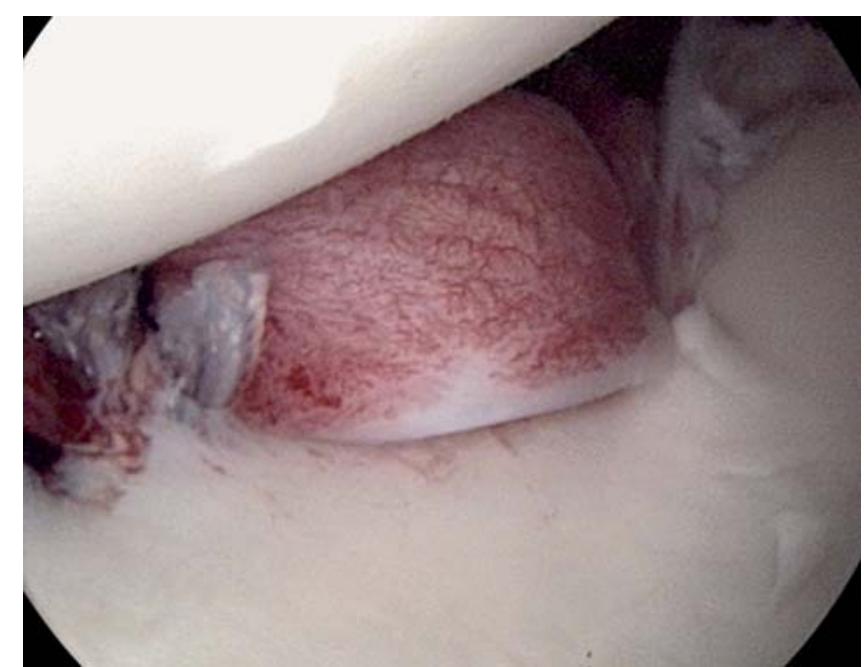

Abb. 5 Arthroskopischer Befund nach Labrumrefixation und inferiorem Kapselshift.

parkt" und anschließend über den anterioren Zugang ausgeleitet. Die Fixierung kann mit speziellen, fadenlosen Ösenankern erfolgen, die in „Press-fit“-Technik eingebracht werden und knotenfrei einen hohen Anpressdruck ermöglichen. Weiter kranial können zusätzlich Fadenanker mit Gewinde (resorbierbar) zur Anwendung kommen. Bei pathologisch vergrößertem Kapselvolumen ist häufig additiv ein arthroskopischer inferiorer Kapselshift erforderlich. Die inferiore Kapsel kann durch ein Release am unteren Glenoid mobilisiert und mittels eingebrachter Zugfäden nach kranial „geshiftet" werden. Der Shift kann kontrolliert und dosiert mit einem fadenlosen Ösenanker fixiert werden (Abb. 5).

\section{Nachbehandlung}

Zur Nachbehandlung wird die operierte Schulter tagsüber für 4 Wochen in einer Schulterorthese (z. B. Omomed ${ }^{\circledR}$ ) immobilisiert, um die nicht erlaubten Bewegungsausmaße (Abduktion und Anteversion über $90^{\circ}$ ) $\mathrm{zu}$ verhindern. Die Außenrotation ist in dieser Zeit vollständig untersagt. Nachts wird die Schulter in einer rigideren Orthese (z.B. Armfix ${ }^{\circledR}$ ) ruhig gestellt. Bereits am 1. postoperativen Tag wird mit einer krankengymnastischen Beübung begonnen, welche die o.g. Limitationen berücksichtigen. Vor Wiederaufnahme eines sportlichen Trainings (in der Regel nach 3-6 Monaten) muss das Muskelaufbautraining abgeschlossen sein. Hierzu sollte insbesondere bei aktiven Sportlern der Trainingszustand mit einem objektiven Messverfahren (z.B. Cybex ${ }^{\circledR}$ ) überprüft werden. Unseres Erachtens hat es sich bewährt, den Patienten sowie den weiterbehandelnden Kollegen ein standardisiertes Nachbehandlungsschema in schriftlicher Form an die Hand zu geben.

\section{Schulterluxation mit begleitender Fraktur des Tuberculum majus}

Die Schulterluxation mit begleitender Fraktur des Tuberculum majus stellt einen Sonderfall dar. Dieses Verletzungsmuster wird in ca. $10 \%$ der Erstluxationen beobachtet [5]. Die Rezidivrate einer Schulterreluxation ist hierbei deutlich erniedrigt.

Eine Reluxation wird nur in ca. $8 \%$ der Fälle beobachtet.

Eine operative Schulterstabilisierung ist somit nur in Ausnahmefällen erforderlich. Die Therapie der Fraktur des Tuberculum majus hängt ab vom Ausmaß der Dislokation. Diese lässt sich meist nur sicher in der CT beurteilen. In der Literatur ist die Indikation zur Reposition und Osteosynthese bei einer Dislokation von mehr als $10 \mathrm{~mm}$ gegeben [11]. Bei einem Hochstand des Tuberculum majus geht der Trend zu einer eher strengeren Indikationsstellung ( $5 \mathrm{~mm}$ ), da ein knöchernes Impingement aufgrund eines in Fehlstellung konsolidierten Tuberculum majus nur schwierig behandelbar ist. Abhängig vom Frakturtyp kann eine Schraubenosteosynthese oder eine Fadenzuggurtungs-Osteosynthese erfolgen. Die Fadenzuggurtung bringt den Vorteil mit sich, dass die Zug- und Rotationskräfte der Rotatorenmanschette auf das refixierte Tuberculum majus neutralisiert werden [12].

\section{Literatur}

1 Steinbruck K. Epidemiology of sports injuries - 25-year-analysis of sports orthopedictraumatologic ambulatory care. Sportverletz Sportschaden 1999; 13: 38 - 52

2 Spencer Jr EE. Treatment of grade III acromioclavicular joint injuries: a systematic review. Clin Orthop Relat Res 2007; 455: 38 - 44

${ }^{3}$ Larsen E, Bjerg-Nielsen A, Christensen P. Conservative or surgical treatment of acromioclavicular dislocation. A prospective, controlled, randomized study. J Bone Joint Surg [Am] 1986; 68: $552-555$

4 Wojtys EM, Nelson G. Conservative treatment of Grade III acromioclavicular dislocations. Clin Orthop Relat Res 1991; 268: 112 - 119

${ }^{5}$ Hovelius L, Olofsson A, Sandstrom B, Augustini $B G$, Krantz L, Fredin H, Tillander B, Skoglund $U$, Salomonsson B, Nowak J, Sennerby U. Nonoperative treatment of primary anterior shoulder dislocation in patients forty years of age and younger, a prospective twentyfive-year follow-up. J Bone Joint Surg [Am] 2008; 90: 945-952

${ }^{6}$ Rowe CR, Patel D, Southmayd WW. The Bankart procedure: a long-term end-result study. J Bone Joint Surg [Am] 1978; 60: 1-16

7 Itoi E, Hatakeyama Y, Sato T, Kido T, Minagawa $H$, Yamamoto N, Wakabayashi I, Nozaka K. Immobilization in external rotation after shoulder dislocation reduces the risk of recurrence. A randomized controlled trial. J Bone Joint Surg [Am] 2007; 89: 2124 - 2131

8 Sudkamp NP, Granrath M, Hoffmann R, Haas $N P$. Instability of the shoulder joint in the athlete. Chirurg 1994; 65: 901 - 909

9 Bottoni CR, Smith EL, Berkowitz MJ, Towle RB, Moore JH. Arthroscopic versus open shoulder stabilization for recurrent anterior instability: a prospective randomized clinical trial. Am J Sports Med 2006; 34: 1730 - 1737

10 Sachs RA, Williams B, Stone ML, Paxton L, Kuney $M$. Open Bankart repair: correlation of results with postoperative subscapularis function. Am J Sports Med 2005; 33: 1458 1462

${ }^{11}$ Neer 2nd CS. Displaced proximal humeral fractures: part I. Classification and evaluation. 1970. Clin Orthop Relat Res 2006; 442: 77-82

12 Bhatia DN, van Rooyen KS, du Toit DF, de Beer $J F$. Surgical treatment of comminuted, displaced fractures of the greater tuberosity of the proximal humerus: a new technique of double-row suture-anchor fixation and long-term results. Injury 2006; 37: 946-952

Prof. Dr. med. Norbert P. Südkamp

Geschäftsführender Direktor

Dr. med. Dirk Maier

Assistenzarzt

Dr. med. Martin Jaeger

Oberarzt

Priv.-Doz. Dr. med. Wolfgang Köstler Leitender Oberarzt, stellvertretender Chefarzt

Department für Orthopädie und

Traumatologie

Universitätsklinikum der AlbertLudwigs-Universität Freiburg

Hugstetter Straße 55

79106 Freiburg 